\title{
The articular joint and its intriguing gliding surfaces
}

\author{
Mats Brittberg
}

Published online: 6 April 2014

(C) Springer-Verlag Berlin Heidelberg 2014

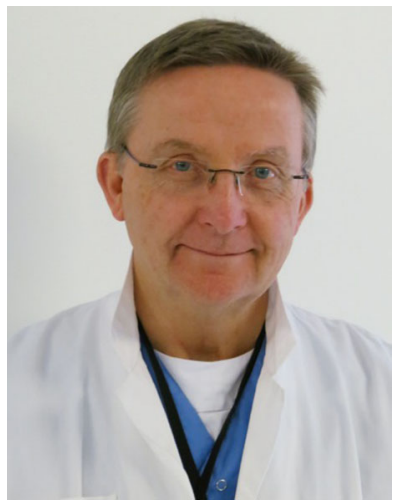

In 1969, the first man made his first steps on the moon. It was a fantastic research work behind that enormous achievement. Ten years earlier, in 1959, Pridie described how to treat cartilage lesions with bare bone with multiple drilling. Eighteen years after the first moon walk, chondrocytes were used to treat cartilage lesions for the first time. Many of us looking at the moon walks expected a large future of space invasions with a living on the moon but it has not yet happened. Similarly, many believed that cell implantations quite fast would heal OA joints, but that has also not happened. However, looking at details, space research has led to an enormous amount of innovations that facilitates daily life. Also, in cartilage research, we have many small research pieces that all together start to make the cartilage puzzle's image slightly visible even if it is still partly blurred.

\section{Brittberg $(\square)$}

Consultant Orthopaedic Surgeon, Region Halland Orthopaedics,

Kungsbacka Hospital, University of Gothenburg,

43480 Kungsbacka, Sweden

e-mail: Mats.Brittberg@regionhalland.se
In this volume, we have the pleasure to present a large number of cartilage-related papers. Most of them are clinically related but some are purely basic in science. The different papers have been put into subcategories. There is one RCT paper to pay some attention to. Still in orthopaedics, there are too few numbers of RCT: $s$ means that every new one published could give us valuable knowledge. The number of patients in the study was unfortunately low, only 25 patients with 11 treated by microfracture and 14 with mosaicplasty plugs and followed for 9.8 years and with equal clinical results. As with a low number of patients, one always has to discuss about the statistical power.

The disease osteochondritis dissecans has intrigued many doctors, and there are many theories of the aetiology. The possibility to treat our young patients with this condition is important, and updates are needed at least every fifth year related to the development in the research field of tissue engineering and imaging. A review paper on OCD included in this cartilage issue will give us the latest updates to tell us how we in the second decade of the new century could treat our OCD patients. One paper is also included in the treatment of osteochondral injuries of the elbow. With a lot of more violent sports appearing, also the smaller joints will be more present in the trauma spheres and the elbow is increasing in the reports of frequencies related to osteochondral injuries.

Two large sections are for clinical repairs in the knee and in the ankle. We could learn from those papers that there are a large variety of new materials used to augment the repair using primarily one stage bone marrow derived techniques with osteochondral plugs some of them as biomimetic scaffolds with multilayered constructs. However, also the cell-based tissue-engineered implants are presented from first generation of ACI to osteochondral implants. 
To evaluate the results after repair surgery, one needs reliable tools. Subsequently, six papers are included presenting different ways to use imaging for a quality assessment. Of special interest is a paper on the biomechanical evaluation of the repair cartilage by means of quantitative T2 imaging. Another section looks at the postoperative appearances of the repair tissue and surrounding tissues describing repair tissue hypertrophy and subchondral bone oedema, two signs that may endanger the repair results postoperatively.

A substantial part of a successful cartilage repair is the postoperative management and weight bearing. Still in 2014, there is not enough science behind different rehab protocols used for patients after cartilage repair operations. However, we could include two papers on joint activities after the operation and in running. Of extra interest is the paper presenting a study on marathon runners. Ten knee asymptomatic marathon beginners underwent a supervised 6-month training programme, which was finalized by the participation at a marathon run. A quantitative MRI was done before the training programme and 1 day after the marathon. The authors found that high-impact forces induced to the joints during a long-distance running were well tolerated also in marathon beginners and did not lead to clinical relevant cartilage loss. In the other study, weight bearing was limited to $20 \mathrm{~kg}$ body weight for 6 weeks after an osteochondral matrix repair. Graft hypertrophy was seen in patients operated on quite acute or in osteochondral defect repairs such as OCD. This is of interest as graft hypertrophy is mostly seen after ACI first generation and weight bearing may induce such a hypertrophy. In this paper, the weight bearing was restricted but other factors seemed to be involved in the graft hypertrophy development.

Furthermore, there is a paper on the use of hyaluronic acid for knee OA and a paper on the controversial use of PRP for augmentation of a bone marrow stimulation repair.

All biological constructs need a strong base to survive for a long time. A reliable cartilage treatment subsequently needs to be secured by strong basic science. In this special issue on cartilage, we have included 4 basic science papers of interest. Of special interest is the paper studying whether bottoming of osteochondral grafts may prevent graft subsidence in the case of an early weight bearing or loading. The authors' results indicate that in a clinical setting, it is advisable to place grafts bottomed, as this may allow for the earlier postoperative mobilization because of the decreased chance of graft subsidence.

Finally, if you spare some time to sit down and go through the cartilage papers in this issue, you may add valuable knowledge about cartilage and cartilage repair to your tool box, and some of the ideas and results presented may also become of benefit for the cartilage traumatized patient.

Enjoyable reading!

\section{Mats Brittberg}

